EPJ Web of Conferences 38, 06001 (2012)

DOI: $10.1051 /$ epjconf/20123806001

(C) Owned by the authors, published by EDP Sciences, 2012

\title{
Gamow-Teller resonances and a separable approximation for Skyrme tensor interactions
}

\author{
A. P. Severyukhin ${ }^{1, a}$ and H. Sagawa ${ }^{2,3}$ \\ 1 Bogoliubov Laboratory of Theoretical Physics, Joint Institute for Nuclear Research, 141980 Dubna, Russia \\ 2 Center for Mathematics and Physics, University of Aizu, Aizu-Wakamatsu, Fukushima 965-8560, Japan \\ 3 RIKEN, Nishina Center, Wako, 351-0198, Japan
}

\begin{abstract}
A finite rank separable approximation for the quasiparticle random phase approximation (QRPA) with Skyrme interactions is applied to study properties of the Gamow-Teller (GT) resonances in the neutronrich $\mathrm{Cd}$ isotopes. This approximation enables one to reduce considerably the dimension of matrix that must be diagonalized to perform QRPA calculations in a very large configuration space. Our results from the SGII Skyrme interaction with the tensor interactions and the density-dependent zero-range pairing interaction show that the GT distribution is noticeably modified when the tensor correlations are taken into account. In particular, for ${ }^{130} \mathrm{Cd}$ the dominant peak is moved 3.6 MeV downward and $10 \%$ of the GT distribution is shifted to the high excitation energy region near $\mathrm{E}=50 \mathrm{MeV}$.
\end{abstract}

\section{Introduction}

A study of the properties of Gamow-Teller (GT) states in the neutron-rich nuclei is an interesting problem not only from the nuclear structure point of view but also from the nuclear astrophysics applications. One of the successful tools for the studies of GT strength distributions is the quasiparticle random phase approximation (QRPA) with the self-consistent mean-field derived by the Skyrme interaction, see for example [1-5]. These QRPA calculations allow one to relate the properties of the ground states and excited states through the same energy density functional.

On the other hand, the experimental studies using the multipole decomposition analysis of the $(n, p)$ and $(p, n)$ reactions $[6,7]$ have clarified the longstanding problem of the missing experimental GT strength, hence resolving the discrepancy between the RPA predictions and GT measurements. It would be desirable to extend the description beyond the QRPA scheme in order to include damping effects observed experimentally [8-10]. Moreover, it is necessary to take into account the tensor correlations that results in shifting some strength up to the higher energy region $[8$, 10,11]. Using the Skyrme functional within the RPA, such attempts in the past $[12,13]$ allow one to understand the damping of charge-exchange resonances and their particle decay. Recently, the damping of the GT mode was investigated using Skyrme RPA plus particle-vibration coupling [14]. However, the size of the configuration space increases very rapidly and one has to study only a limited number of typical cases. It would be helpful to have an approach where the size of the calculations does not depend directly on the size of the configuration space.

Making use of separable residual interaction one can perform calculations in a large configuration space since there is a way to reduce the dimension which grow with the size of configuration space [15]. For the same reason, the

\footnotetext{
a e-mail: sever@theor.jinr.ru
}

finite rank separable approximation (FRSA) for the QRPA with Skyrme interactions [16-18] has been extended to describe of charge-exchange excitation modes [19]. In the case of the FRSA the determination of the QRPA eigenenergies requires to calculate the zeros of a determinant whose dimension does not depend on the size of the twoquasiparticle configuration space. Before to investigate the effect of the $2 \mathrm{p}-2 \mathrm{~h}$ fragmentation one needs to be sure that the FRSA is good enough to reproduce the characteristics of spin-isospin excitations. The method has been validated in the case of the ${ }^{90} \mathrm{Zr}$ and ${ }^{132} \mathrm{Sn}$ regions by using the FRSA to calculate the GT and spin-dipole strength distributions in Tamm-Dancoff approximation (TDA), and comparing them with HF-TDA results obtained without the separable approximation $[19,20]$. The approach is generalized to take into account the QRPA tensor correlations [21].

This paper gives an illustration of the approach for the GT strength distributions of the neutron-rich $\mathrm{Cd}$ isotopes.

\section{Method of calculation}

The starting point of the method is the HF-BCS calculation [22] of the parent ground state, where the spherical symmetry is imposed on the quasiparticle wave functions. In the particle-hole $(\mathrm{p}-\mathrm{h})$ channel we use the Skyrme interaction with the triplet-even and triple-odd tensor terms which were proposed in the pioneering works [23,24]. The continuous part of the single-particle spectrum is discretized by diagonalizing the HF hamiltonian on a harmonic oscillator basis. The inclusion of the tensor terms results in the following modification of the spin-orbit potential in coordinate space $[11,25]$ :

$$
U_{\text {S.O. }}^{(q)}=\frac{W_{0}}{2 r}\left(2 \frac{d \rho_{q}}{d r}+\frac{d \rho_{q^{\prime}}}{d r}\right)+\left(\alpha \frac{J_{q}}{r}+\beta \frac{J_{q^{\prime}}}{r}\right),
$$

where $\rho_{q}$ and $J_{q}(q=n, p)$ are the densities and the spinorbit densities, respectively. The values $\alpha$ and $\beta$ can be sep- 
arated into contributions of the central force $\left(\alpha_{c}, \beta_{c}\right)$ and the tensor force $\left(\alpha_{T}, \beta_{T}\right)[11,25]$.

We work in the quasiparticle representation defined by the canonical Bogoliubov transformation:

$$
a_{j m}^{+}=u_{j} \alpha_{j m}^{+}+(-1)^{j-m} v_{j} \alpha_{j-m},
$$

where $\alpha_{j m}^{+}\left(\alpha_{j m}\right)$ is the quasiparticle creation (annihilation) operator and $j m$ denote the quantum numbers $n l j m$. The pairing correlations are generated by the density-dependent zero-range force

$$
V_{\text {pair }}\left(\mathbf{r}_{1}, \mathbf{r}_{2}\right)=V_{0}\left(1-\eta\left(\frac{\rho\left(r_{1}\right)}{\rho_{0}}\right)^{\gamma}\right) \delta\left(\mathbf{r}_{1}-\mathbf{r}_{2}\right) .
$$

The parameter $\rho_{0}$ is equal to the nuclear saturation density, $V_{0}, \eta$ and $\gamma$ are fixed to reproduce the odd-even mass difference of corresponding nuclei.

The $\mathrm{p}-\mathrm{h}$ residual interaction can be obtained as the second derivative of the energy density functional with respect to the densities. The main simplification is to replace the central p-h interaction $V_{p h}^{C}$ by its Landau-Migdal approximation and to keep only the $l=0$ terms (all Landau parameters with $l>1$ are zero). The expressions for the Landau parameters in terms of the Skyrme force parameters can be found in Ref. [26]. Also, the Coulomb residual interaction is dropped. Therefore we can write $V_{p h}^{C}$ in the spin-isospin channel as

$$
\begin{array}{r}
V_{p h}^{C}=\tau^{(1)} \tau^{(2)} N_{0}^{-1} G_{0}^{\prime}\left(r_{1}\right) \sigma^{(1)} \cdot \sigma^{(2)} \delta\left(\mathbf{r}_{1}-\mathbf{r}_{2}\right) \\
=\tau^{(1)} \tau^{(2)} N_{0}^{-1} \frac{G_{0}^{\prime}\left(r_{1}\right)}{r_{1}^{2}} \delta\left(r_{1}-r_{2}\right) \\
\quad \times \sum_{J M} \sum_{L=J ; J \pm 1} T_{L J M}\left(\hat{r}_{1}, \sigma_{1}\right) T_{L J M}^{*}\left(\hat{r}_{2}, \sigma_{2}\right),
\end{array}
$$

where $\sigma^{(i)}$ and $\tau^{(i)}$ are the spin and isospin operators, $T_{L J M}(\hat{r}, \sigma)=\left[Y_{L} \times \sigma\right]_{J}^{M}$, and $N_{0}=2 k_{F} m^{*} / \pi^{2} \hbar^{2}$ with $k_{F}$ and $m^{*}$ standing for the Fermi momentum and nucleon effective mass. Let us explain the FRSA for the $V_{p h}^{C}$ matrix elements. The central p-h interaction that is given by Eq. (4) is in the separable form in the angular coordinates. The radial integrals can be calculated accurately by choosing a large enough cutoff radius $R$. By means of a $N$-point integration Gauss formula with abscissas $r_{k}$ and weights $w_{k}$, the $V_{p h}^{C}$ matrix elements can be written as a sum of $N$ terms [16, 19]. The value $N=45$ is sufficient for the desired accuracy of description of the spin-isospin excitations [19].

We simplify the tensor $\mathrm{p}$-h interaction by replacing it by the two-term separable interaction as introduced in [27],

$$
\begin{gathered}
V_{p h}^{T}\left(\mathbf{r}_{1}, \mathbf{r}_{2}\right)=V_{T 1}\left(\mathbf{r}_{1}, \mathbf{r}_{2}\right)+V_{T 1}\left(\mathbf{r}_{2}, \mathbf{r}_{1}\right)+V_{T 2}\left(\mathbf{r}_{1}, \mathbf{r}_{2}\right), \\
V_{T 1}=\tau^{(1)} \tau^{(2)} \lambda_{1} \sum_{M} T_{01 M}\left(\hat{r}_{1}, \sigma_{1}\right) r_{2}^{2} T_{21 M}^{*}\left(\hat{r}_{2}, \sigma_{2}\right), \\
V_{T 2}=\tau^{(1)} \tau^{(2)} \lambda_{2} \sum_{M} r_{1}^{2} T_{21 M}\left(\hat{r}_{1}, \sigma_{1}\right) r_{2}^{2} T_{21 M}^{*}\left(\hat{r}_{2}, \sigma_{2}\right),
\end{gathered}
$$

where the strengths $\lambda_{1}$ and $\lambda_{2}$ are adjusted to reproduce the centroid energies of the GT and spin-quadrupole strength distributions calculated with the original tensor $\mathrm{p}-\mathrm{h}$ interaction.

We introduce the phonon creation operators

$$
\begin{gathered}
Q_{J M i}^{+}=\sum_{j_{n} j_{p}}\left(X_{j_{n} j_{p}}^{J i} A^{+}\left(j_{n} j_{p} ; J M\right)\right. \\
\left.-(-1)^{J-M} Y_{j_{n} j_{p}}^{J i} A\left(j_{n} j_{p} ; J-M\right)\right), \\
A^{+}\left(j_{n} j_{p} ; J M\right)=\sum_{m_{n} m_{p}}\left\langle j_{n} m_{n} j_{p} m_{p} \mid J M\right\rangle \alpha_{j_{n} m_{n}}^{+} \alpha_{j_{p} m_{p}}^{+},
\end{gathered}
$$

where the index $J$ denotes total angular momentum and $M$ is its z-projection in the laboratory system. One assumes that the ground state is the phonon vacuum $|0\rangle$ and the excited states are $Q_{J M i}^{+}|0\rangle$ with the normalization condition

$$
\sum_{j_{n} j_{p}} X_{j_{n} j_{p}}^{J i} X_{j_{n} j_{p}}^{J i^{\prime}}-Y_{j_{n} j_{p}}^{J i} Y_{j_{n} j_{p}}^{J i^{\prime}}=\delta_{i i^{\prime}}
$$

Making use of the linearized equation-of-motion approach one can get the QRPA equations [22]:

$$
\left(\begin{array}{ll}
\mathcal{A} & \mathcal{B} \\
-\mathcal{B} & -\mathcal{A}
\end{array}\right)\left(\begin{array}{l}
X \\
Y
\end{array}\right)=\omega\left(\begin{array}{l}
X \\
Y
\end{array}\right) .
$$

Solutions of this set of linear equations yield the eigenenergies and the amplitudes $X, Y$ of the excited states. The dimension of the matrices $\mathcal{A}, \mathcal{B}$ is the size of the twoquasiparticle configuration space. One can find a prescription how the FRSA and the separable form of the tensor $\mathrm{p}$-h interaction (5) can simplify the solution of the QRPA equations in Ref. [21]. The QRPA equations (11) can be reduced to the secular equation and the matrix dimension never exceeded $(4 N+4) \times(4 N+4)$ irrespective to the configuration space size. If we omit the tensor $\mathrm{p}-\mathrm{h}$ interaction (5) then the maximum dimension is $4 N \times 4 N$ [19]. The approach enables one to obtain the eigen-energies $\omega_{J i}$ as the roots of the secular equation and the phonon amplitudes $X_{j_{n} j_{p}}^{J i}$ and $Y_{j_{n} j_{p}}^{J i}$ can be calculated by performing the partial summations of the secular matrix [19]. The excitation energies with respect to the parent ground state are given by

$$
E_{J i}^{\mp}=\omega_{J i} \mp\left(\mu_{n}-\mu_{p}\right)
$$

in both the $T_{\mp}$ channels. The quantities $\mu_{q}$ are the chemical potentials.

As an application of the method we have studied the GT strength distributions of the parent nuclei ${ }^{126,128,130} \mathrm{Cd}$.

\section{Application to ${ }^{126-130} \mathrm{Cd}$}

As the parameter set in the particle-hole channel, we use the central Skyrme interaction SGII [26] and the same zerorange tensor interaction as that in the paper [27]. The SGII parametrization is successful describing the spin-dependent properties as the central Skyrme force. In particular, one obtains a good description of experimental energies of the GT resonance of ${ }^{90} \mathrm{Zr}$. The single-particle continuum is discretized by diagonalizing the HF hamiltonian on a basis of 12 harmonic oscillator shells and cutting off the singleparticle spectra at the energy of $100 \mathrm{MeV}$. This is sufficient to exhaust the Ikeda sum rule $3(N-Z)$ [28] as well as the sum rule for the spin-dipole strength $[29,30]$. 


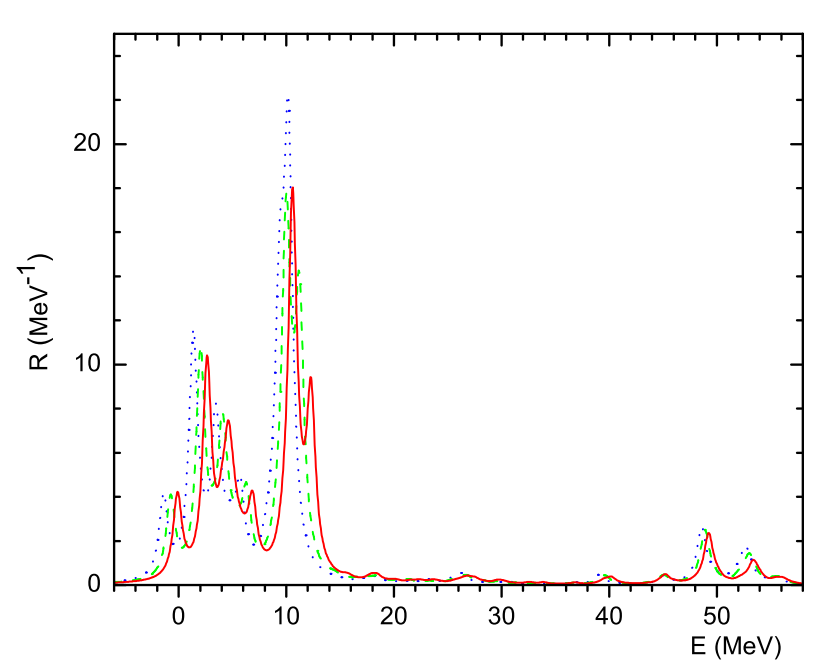

Fig. 1. The GT strength distributions in $\sigma \tau_{-}$channel of ${ }^{126} \mathrm{Cd}$ (solid line), ${ }^{128} \mathrm{Cd}$ (dashed line), ${ }^{130} \mathrm{Cd}$ (dotted line).

We use the isospin-invariant surface-peaked pairing force (3), with $\eta=1, \alpha=1$ and the value $\rho_{0}=0.16 \mathrm{fm}^{-3}$ for the nuclear saturation density. The strength $V_{0}$ is taken to be $-870 \mathrm{MeV} \mathrm{fm}^{3}$ to get a reasonable description of the experimental pairing energies of ${ }^{126,128} \mathrm{Cd}$ [19]. The definition of the pairing force (3) involves the energy cutoff of the single-particle space to restrict the active pairing space. We use the soft cutoff at $10 \mathrm{MeV}$ above the Fermi energies as proposed in Refs. [17,31].

The Landau parameters expressed in terms of the Skyrme force parameters depend on $k_{F}$ [26]. To study the properties of the electric excitations one can adopt some effective value for $k_{F}$ to give an accurate representation of the central p-h Skyrme interaction [16,32]. In this work, the nuclear matter value for $k_{F}$ is used. This means that we don't change the original value $G_{0}^{\prime}=0.50$ (at saturation density) which plays the important role of determining the characteristics of the GT states [26]. As the parameter set of the tensor $\mathrm{p}$-h interaction (5) we assume the following values

$$
\begin{aligned}
& \lambda_{1}=\frac{1300}{A^{2}} \mathrm{MeV} \cdot \mathrm{fm}^{-2}, \\
& \lambda_{2}=\frac{36}{A^{2}} \mathrm{MeV} \cdot \mathrm{fm}^{-4} .
\end{aligned}
$$

These parameter values in the case of the nuclei ${ }^{90} \mathrm{Zr}$ and ${ }^{208} \mathrm{~Pb}$ lead to the same main features of GT and spin-quadrupole strength distributions compared with the exact ones [27]. It is worth mentioning that the $\lambda_{2}$ term of the interaction (5) weakly affects the GT strength distributions. All calculations are without any quenching factor. In the figures, the calculated strength distributions are folded out with a Lorentzian distribution of $1 \mathrm{MeV}$ width. The excitation energies refer to the ground state of the parent nucleus.

First, we examine the role of the tensor interactions on QRPA calculations. Effects of this component of the Skyrme interaction on the Ikeda sum rules of ${ }^{126,128,130} \mathrm{Cd}$ are shown in Table 1. One can see that the tensor correlations shift about $10 \%$ of the GT strength from the lower energy region $(E \leq 30 \mathrm{MeV})$ to the higher energy region. As a result, about $90 \%$ of the GT strength distribution is located below $30 \mathrm{MeV}$ with respect to the parent ground state. At the same time the effects lead to an increase of

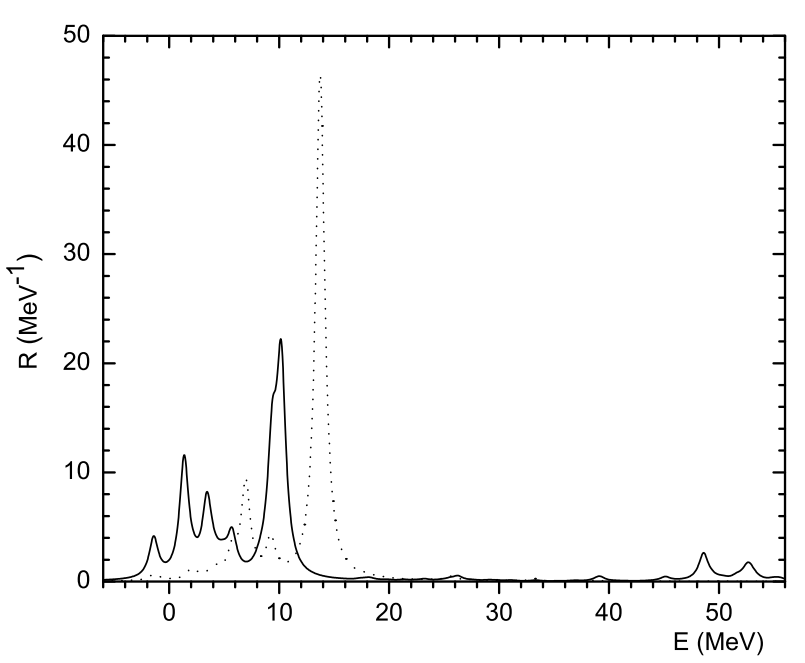

Fig. 2. Effects of tensor correlations on the GT strength distribution in $\sigma \tau_{-}$channel of ${ }^{130} \mathrm{Cd}$. Solid and dotted lines correspond to calculations with and without the tensor terms, respectively.

Table 1. Ikeda sum rule values of GT states. $S_{-}, S_{+}$are the summed strength values of the $\sigma \tau_{-}$and $\sigma \tau_{+}$channels, respectively. $\Delta S_{\mp}$ correspond to the summed strength values below $30 \mathrm{MeV}$.

\begin{tabular}{llllllc}
\hline & & $\Delta S_{-}$ & $S_{-}$ & $\Delta S_{+}$ & $S_{+}$ & $S_{-}-S_{+}$ \\
\hline${ }^{126} \mathrm{Cd}$ & no tensor & 89.6 & 90.6 & 0.6 & 0.7 & 89.9 \\
& with tensor & 84.1 & 93.6 & 3.3 & 3.6 & 90.0 \\
${ }^{128} \mathrm{Cd}$ & no tensor & 95.6 & 96.6 & 0.4 & 0.6 & 96.0 \\
& with tensor & 89.1 & 99.4 & 3.2 & 3.5 & 95.9 \\
& & & & & & \\
${ }^{130} \mathrm{Cd}$ & no tensor & 101.5 & 102.5 & 0.3 & 0.5 & 102.0 \\
& with tensor & 94.3 & 105.5 & 3.2 & 3.5 & 102.0 \\
\hline
\end{tabular}

both $S_{-}$and $S_{+}$sum rules keeping the difference $S_{-}$and $S_{+}$the same. It is noticed in figures 1,2 and Table 1 that the tensor interactions change the contributions in separate $\sigma \tau_{-}$and $\sigma \tau_{+}$channels. For the $\sigma \tau_{+}$channel, the Pauli blocking of the neutron excess is the reason why the $S_{+}$ value is much smaller than the $S_{-}$value. As can be seen from Table 1 , the $\sigma \tau_{+}$channel is more sensitive to the inclusion of the tensor terms in the absolute magnitude.

One can expect an increase of the high energy strength if the coupling of the $1 \mathrm{p}-1 \mathrm{~h}$ configurations to more complex configurations is taken into account. The computational developments that would allow us to study this point are still underway.

The sum rule is an integral characteristic and it is less sensitive to the details than the strength distribution. Figure 1 shows the evolution of the GT strength distributions. When the tensor terms are not included, for ${ }^{126,128,130} \mathrm{Cd}$ the GT strength distributions calculated with the same parameter set are rather well studied in Ref. [19]. In this work our results indicate a noticeable redistribution of the GT strengths that were calculated with the tensor interaction. As an illustration we show in figure 2 the GT strengths of ${ }^{130} \mathrm{Cd}$ calculated with and without the tensor interaction. One can see that the tensor correlations induces 3.6 $\mathrm{MeV}$ downward shift of the main peak in this nucleus. Also, part of the main peak strength is fragmented in the low-energy peaks and also a high-energy tail is formed at 
around $\mathrm{E}=50 \mathrm{MeV}$. Looking at the main peak we find that the main configuration is $\left\{\pi 1 h \frac{9}{2} \nu 1 h \frac{11}{2}\right\}(80 \%$ and $64 \%$ of the QRPA calculations with and without the tensor terms, respectively).

As pointed out in Ref. [19] the effect of pairing on the GT states vanishes in the case of ${ }^{130} \mathrm{Cd}$ which has $\mathrm{N}=82$ and therefore, no neutron pairing correlations can be effective. In particular, for ${ }^{130} \mathrm{Cd}$ the GT strength distributions calculated with and without the pairing coincide in the resonance energy interval. The main reason is the large neutron excess. To see this, we consider again the structure of the main peak in the case of ${ }^{130} \mathrm{Cd}$. The largest contribution to the wave function comes from the two-quasiparticle configuration $\left\{\pi 1 h \frac{9}{2} v 1 h \frac{11}{2}\right\}$, where proton subshell $1 h \frac{9}{2}$ is out of the proton-pairing window. It is worth mentioning that the RPA calculations are made by the filling approximation [22].

\section{Summary}

Starting from the Skyrme SGII+T mean-field calculations, properties of the Gamow-Teller resonances in the neutronrich $\mathrm{Cd}$ isotopes are analyzed in the framework the finite rank separable approach for the QRPA calculations taking into account the tensor correlations. The present approach enables one to perform the calculations in very large configuration spaces without spending too much computer time.

At a qualitative level, we observe that this component of the interaction is important for predictions for the GT strength distributions. The inclusion of the tensor correlations leads to a redistribution of the GT strength. In addition, there is an increase of the sum rule strengths in both $\sigma \tau_{-}$and $\sigma \tau_{+}$channels. It is shown that the tensor correlations shift up $10 \%$ of the total GT strength to the higher energy region. Also, part of the main peak strength is fragmented in the low-energy peaks and main peak is moved downwards. In particular, for ${ }^{130} \mathrm{Cd}$ this shift is large as much as $3.6 \mathrm{MeV}$.

The effects of pairing on the GT states are important in these nuclei, and a QRPA approach is thus appropriate. There is only the visible neutron-pairing effect on the GT resonances. The strong difference in the neutron and proton chemical potentials plays the key role to explain this peculiarity [19].

The FRSA allows one to simplify the evaluation of the coupling of QRPA phonons to more complex configurations and to calculate thus the fragmentation and damping of the QRPA excitations. This study is now in progress.

\section{Acknowledgments}

We are grateful to Prof. V. V. Voronov and Prof. Nguyen Van Giai for useful discussions. A.P.S. thank the hospitality of IPN-Orsay where a part of this work was done. This work is partly supported by the IN2P3-RFBR agreement No. 110291054.

\section{References}

1. J. Engel, M. Bender, J. Dobaczewski, W. Nazarewicz, R. Surman, Phys. Rev. C 60, 014302 (1999)
2. M. Bender, J. Dobaczewski, J. Engel, W. Nazarewicz, Phys. Rev. C 65, 054322 (2002)

3. S. Fracasso, G. Colò, Phys. Rev. C 76, 044307 (2007)

4. C. L. Bai, H. Sagawa, H. Q. Zhang, X. Z. Zhang, G. Colò, F. R. Xu, Phys. Lett. B 675, 28 (2009)

5. C.L. Bai, H.Q. Zhang, H. Sagawa, X.Z. Zhang, G. Colò, F.R. Xu, Phys. Rev. C 83, 054316 (2011)

6. T. Wakasa et al., Phys. Rev. C 55, 2909 (1997)

7. M. Ichimura, H. Sakai, T. Wakasa, Prog. Part. Nucl. Phys. 56, 446 (2006)

8. G.F. Bertsch, I. Hamamoto, Phys. Rev. C 26, 1323 (1982)

9. V. A. Kuzmin, V. G. Soloviev, J. Phys. G: Nucl. Phys. 10, 1507 (1984)

10. S. Drozdz, F. Osterfeld, J. Speth, J. Wambach, Phys. Lett. B 189, 271 (1987)

11. G. Colò, H. Sagawa, S. Fracasso, P.-F. Bortignon, Phys. Lett. B 646, 227 (2007); 668, 457(E) (2008)

12. G. Colò, Nguyen Van Giai, P.-F. Bortignon, R.A. Broglia, Phys. Rev. C 50, 1496 (1994)

13. G. Colò, H. Sagawa, Nguyen Van Giai, P.-F. Bortignon, T. Suzuki, Phys. Rev. C 57, 3049 (1998)

14. Y.F. Niu, G. Coló, M. Brenna, P.F. Bortignon, J. Meng, Phys. Rev. C 85, 034314 (2012)

15. V. G. Soloviev, Theory of Atomic Nuclei: Quasiparticles and Phonons (Institute of Physics, Bristol and Philadelphia, 1992)

16. Nguyen Van Giai, Ch. Stoyanov, V.V. Voronov, Phys. Rev. C 57, 1204 (1998)

17. A.P. Severyukhin, V.V. Voronov, Nguyen Van Giai, Phys. Rev. C 77, 024322 (2008)

18. A.P. Severyukhin, V.V. Voronov, Nguyen Van Giai, Eur. Phys. J. A 22, 397 (2004)

19. A.P. Severyukhin, V.V. Voronov, Nguyen Van Giai, Prog. Theor. Phys. 128, 489 (2012)

20. A.P. Severyukhin, N.N. Arsenyev, V.V. Voronov, Nguyen Van Giai, Phys. At. Nucl. 72, 1149 (2009)

21. A. P. Severyukhin, H. Sagawa, in preparation.

22. P. Ring and P. Schuck, The Nuclear Many Body Problem (Springer, Berlin, 1980).

23. T.H.R. Skyrme, Nucl. Phys. 9, 615 (1959)

24. F. Stancu, D.M. Brink, H. Flocard, Phys. Lett. B 68, 108 (1977)

25. T. Lesinski, M. Bender, K. Bennaceur, T. Duguet, J. Meyer, Phys. Rev. C 76, 014312 (2007)

26. Nguyen Van Giai, H. Sagawa, Phys. Lett. B 106, 379 (1981)

27. C.L. Bai, H.Q. Zhang, X.Z. Zhang, F.R. Xu, H. Sagawa, G. Colò, Phys. Rev. C 79, 041301(R) (2009)

28. K. Ikeda, S. Fujii, J.I. Fujita, Phys. Lett. 3, 271 (1963)

29. A. Krasznahorkay et al., Phys. Rev. Lett. 82, 3216 (1999)

30. K. Yako, H. Sagawa, H. Sakai, Phys. Rev. C 74, 051303(R) (2006)

31. S.J. Krieger, P. Bonche, H. Flocard, P. Quentin, M.S. Weiss, Nucl. Phys. A 517, 275 (1990)

32. A.P. Severyukhin, Ch. Stoyanov, V.V. Voronov, Nguyen Van Giai, Phys. Rev. C 66, 034304 (2002) 